\title{
Calculation of Transformer Ratio in Mason's Equivalent Circuit for cMUTs
}

\author{
Selim Olcum, Abdullah Atalar, Hayrettin Köymen and Muhammed N. Senlik \\ Dept. of Electrical and Electronics Engineering \\ Bilkent University \\ Ankara, Turkey 06800 \\ Email: selim@ee.bilkent.edu.tr
}

\begin{abstract}
We present a new method to calculate the transformer ratio of a cMUT in Mason's Equivalent circuit model. The effect of the spring softening capacitance is also included in the analysis. We use the existing turns ratio calculation methods as a starting point to calculate the force-voltage ratio at the secondary of the transformer and the input port of the circuit. We use this ratio and the capacitances in the Mason's circuit to find the actual turns ratio. Different methods are discussed for the calculation of the equivalent circuit parameters. We show that the transformer ratio has a bounded maximum at collapse voltage. We also investigate the effect of electrode size on the transformer ratio. Transformer ratio decreases with decreasing electrode size.
\end{abstract}

\section{INTRODUCTION}

Capacitive micromachined ultrasonic transducers (cMUTs) have been studied for more than a decade now. Although much effort is being spent to new designs and fabrication technologies, accurate characterization of cMUTs has not been completed yet. The efforts spent on modelling of cMUTs include mathematical models, finite element models and equivalent circuit models. For the characterization of cMUTs, the most common approach is using Mason's equivalent circuit [1]. In this work, we present a method for accurate determination of the transformer ratio in vacuum including the effect of spring softening.

\section{CMUT MODEL}

In this work we use Mason's equivalent circuit to characterize the cMUT behavior [1]. Electrical and mechanical parameters of a cMUT are modelled as lumped electrical components in this circuit. In Fig. 1(a), the capacitance $C_{0}$ stands for the shunt input capacitance of the membrane and the series negative capacitance at the electrical port models the spring softening effect of the membrane [2]. $n$ denotes the conversion factor of the ideal transformer. The lumped element $Z_{m}$ at the mechanical port is the mechanical impedance of the membrane [1].

$Z_{m}$ is usually calculated using the Mason's impedance expression, which yields accurate approximation for thin membranes. In this work, we use a simpler, yet an accurate model [3]for all membranes. The frequency range in which the model is valid depends on the order of the model. The model order increases by adding an L-C section for each successive resonance. The mechanical impedance, resulting



(a)

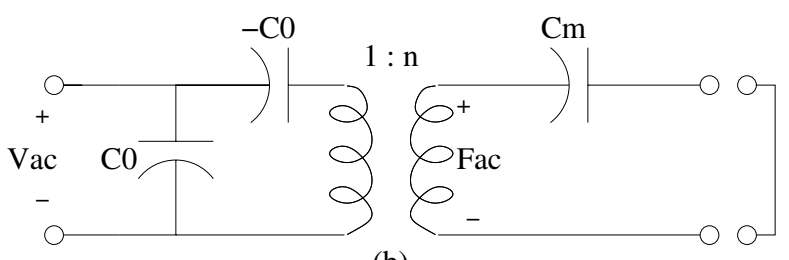

(b)

Fig. 1. (a) Mason's equivalent circuit [1] together with mass spring model. (b) Equivalent circuit for low frequency behavior of a cMUT in vacuum.

from the model in [3], shows very good agreement with Finite Element Method (FEM) simulation results up to the frequency of the highest resonance modelled.

The transformer ratio, $n$ is a property of the electrodynamics of cMUT. It depends on the membrane material, dimensions and applied bias voltage. Note that, transformer ratio is independent of acoustic loading and frequency, in the usual operating range below the first parallel resonance of the membrane.

Consider the first L-C section of the model given in [3] as in Fig. 1(a). Inductive part is insignificant at low frequencies and the model reduces to the circuit in Fig. 1(b). We calculate the ratio of $F_{a c}$ to $V_{a c}$ at DC using small signal approximation, first. Then we correct this ratio using the model in Fig. 1(b) to obtain the transformer ratio, $n$.

\section{PARAMETERS}

\section{A. Mechanical Capacitance, $C_{m}$}

The mechanical capacitance, $C_{m}$ in Fig. 1(b) represents the inverse of the stiffness of the membrane. The value of this capacitance can be calculated precisely using the following formulation as in [3]:

$C_{m}=\left[q_{3}\left(\frac{t_{m}}{a}\right)^{3}+q_{2}\left(\frac{t_{m}}{a}\right)^{2}+q_{1}\left(\frac{t_{m}}{a}\right)+q_{0}\right] \frac{12\left(1-\sigma^{2}\right) a^{2}}{\pi\left(Y_{0}+T\right) t_{m}^{3}}$ 
where $a$ and $t_{m}$ are the radius and the thickness of the membrane, $Y_{0}$ is the Young's modulus, $T$ is the residual stress on the membrane and $\sigma$ is the Poisson's ratio. The value of the polynomial coefficients, $q_{i}$ are given in Table III-A or [3].

Eq. 1 predicts the value of the mechanical capacitance for unbiased cases. However, cMUT should be biased by a DC voltage. Therefore, the mechanical capacitance also changes, since $1 / C_{m}$ represents the stiffness of the membrane. The following formulation as in [3] calculates $C_{m}$ for the biased cases as well:

$C_{m}(\gamma)=C_{m} \times\left[1+0.025 \gamma^{2}\left(1-3.54 \gamma^{2}+7 \gamma^{4}\right)\left(\frac{t_{g}}{t_{m}}\right)^{2}\right]^{-1}$

where $\gamma$ is the ratio of the bias voltage, to the collapse voltage of the membrane $\left(\gamma=V_{d c} / V_{c o l}\right)$ and $t_{g}$ is the gap height. Note that set of Eq. 1 and 2 are valid for different membrane dimensions and for different materials.

The relation between $C_{m}$ and $\kappa$ is given by [1]:

$$
\frac{1}{C_{m}}=\kappa=\frac{16 \pi\left(Y_{0}+T\right) t_{m}^{3}}{\left(1-\sigma^{2}\right) a^{2}}
$$

Note that the inverse of Mason's stiffness approximates the $C_{m}$ values obtained from Eq. 1when the membrane is thin with $t_{m} / a$ ratios less than 0.05 . In addition, if the gap height does not exceed the membrane thickness, the effect of correction in Eq. 2 is less than $10 \%$. Within these limits, we can use Mason's stiffness definition to calculate $C_{m}$ accurately.

\section{B. Deflection Profile, $x(r)$}

We first calculate the deflection profile of the membrane for a given DC bias voltage. This is important for accurate calculations of the electrical parameters of the model. The deflection calculations are based on the method developed for collapse voltage calculations in [4].

In this method, we partition the membrane into force nodes, where the electrostatic force is applied on these nodes. The number of nodes on the membrane effects the accuracy of the results. We used 250 nodes on the membrane for this paper. The deflection for each node is calculated using the expressions in [5]. The resulting deflection profile, $x(r)$ is:

$x(r)=\sum_{i=1}^{N}\left\{\begin{array}{cl}\frac{F_{i}}{8 \pi D}\left[\frac{\left(a^{2}+r^{2}\right)\left(a^{2}-b_{i}^{2}\right)}{2 a^{2}}+\left(b_{i}^{2}+r^{2}\right) \ln \frac{b_{i}}{a}\right], & b_{i}<r ; \\ \frac{F_{i}}{8 \pi D}\left[\frac{\left(a^{2}-r^{2}\right)\left(a^{2}+b_{i}^{2}\right)}{2 a^{2}}+\left(b_{i}^{2}+r^{2}\right) \ln \frac{r}{a}\right], & b_{i} \geq r .\end{array}\right.$

where $F_{i}$ is the electrostatic force between the electrodes at the $i^{t h}$ node, $D$ is the flexural rigidity of the membrane which is equal to, $\frac{\left(Y_{0}+T\right) t_{m}^{3}}{12\left(1-\sigma^{2}\right)}, r$ is the axial distance of the corresponding node to the center node, $b_{i}$ is the axial distance of force $F_{i}$

\begin{tabular}{|c|c|c|c|c|}
\hline & $q_{3}$ & $q_{2}$ & $q_{1}$ & $q_{0}$ \\
\hline$C_{m}$ & -0.007167 & 0.03620 & -0.0005467 & 0.005208 \\
\hline
\end{tabular}

TABLE I

COEFFICIENTS OF EQ. 1 [3].

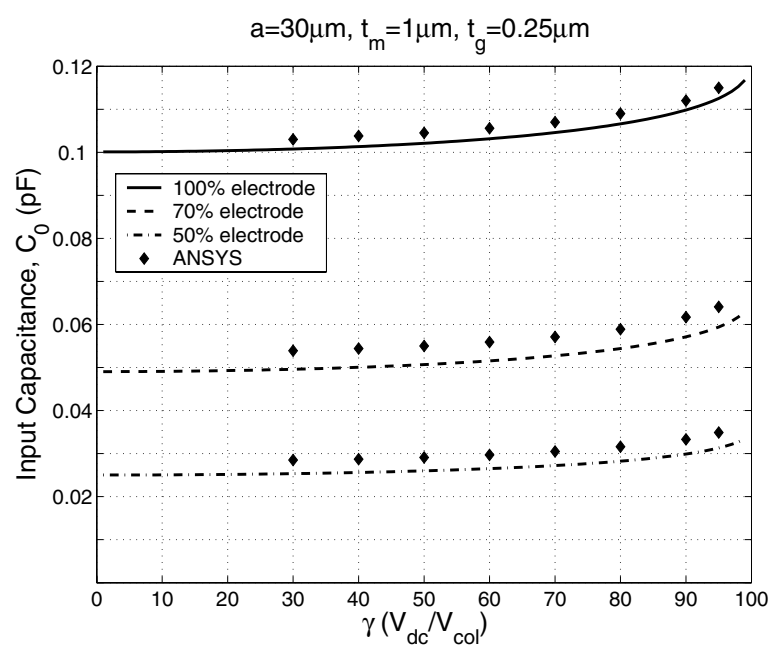

Fig. 2. Calculation of input capacitance with numerical analysis (lines) and FEM simulations (diamonds) as a function of applied bias voltage for three different electrode coverage.

to the center node and $N$ is the total number of nodes. Note that Eq. 4 assumes a thin plate approximation [5].

First, we find the deflection profile using superposition of the forces at each node. Then, we recalculate the electrostatic force in the gap and deflect the membrane again. We iteratively update the gap until the deflection converges. We assume that the membrane deflection is converged when the difference of the two successive iterations is small enough.

\section{Collapse Voltage, $V_{c o l}$}

We calculate the collapse voltage, $V_{c o l}$ of a circular clamped membrane by conducting a line search on a voltage interval. We assume that the collapse voltage is the largest voltage value to make the membrane deflection converge. We obtain good agreement, within $1 \%$, between these calculations and the FEM simulations.

One other simpler yet accurate way of calculating the collapse voltage is using the analytical expression in [6]:

$$
V_{c o l} \simeq K \sqrt{\frac{128\left(Y_{0}+T\right) t_{m}^{3} t_{g}^{3}}{27 \epsilon_{0}\left(1-\sigma^{2}\right) a^{4}}}
$$

where $\epsilon_{0}$ is the free space permittivity and $K$ factor is 0.7 and 0.82 for full and half electrode coverage respectively.

\section{Input Capacitance, $C_{0}$}

Finally, we calculate the shunt input capacitance, $C_{0}$ using the deflection profile found from Eq. 4. We assume that a ring shaped parallel plate capacitor is present between every consecutive force node. The total capacitance is calculated by adding all of these small parallel plate capacitors. We ignore the effect of fringing fields. The effect of fringing fields is more for the case of smaller electrode sizes. The accuracy of the model can be observed in Fig. 2. 


\section{TRANSFORMER RATIO}

In this section we investigate an accurate calculation method for transformer ratio, $n$ If we ignore the effect of the negative spring softening capacitance, $-C_{0}$, the transformer ratio can be defined as in [7]:

$$
n^{\prime}=\left|\frac{\partial F}{\partial V}\right|_{V=V_{d c}}
$$

where $F$ is the electromechanical force acting on the membrane. However such an approach is not feasible if we use coupled field analysis tools of FEM simulators, since the effect of the spring softening capacitance is already included. We note that the spring softening is a coupled field effect. Therefore, for both AC or DC operations, spring softening capacitance should be taken into account. The ratio, $F_{a c} / V_{a c}$ in Fig. 1 is not the transformer ratio when we include the effect of spring softening into our analysis. We call this ratio $n^{\prime}$ and utilize it to determine the transformer ratio, $n$.

The transformer ratio is defined as the ratio of the force on the secondary of the transformer to the voltage on the primary of the transformer. Therefore, we take into account the voltage division because of the spring softening capacitance, $-C_{0}$ :

$$
\begin{aligned}
\frac{F_{a c} / n}{v_{a c}}=\frac{\frac{1}{j \omega n^{2} C_{m}}}{\frac{1}{j \omega}\left(\frac{1}{n^{2} C_{m}}-\frac{1}{C_{0}}\right)} & =\frac{1}{1-n^{2} C_{m} / C_{0}} \\
\frac{F_{a c}}{v_{a c}} & =n^{\prime}=\frac{n}{1-n^{2} C_{m} / C_{0}}
\end{aligned}
$$

Note that Eq. 7 is independent of the signal frequency. Therefore, the solution of this equation is also valid for $\mathrm{AC}$ calculations provided that the solution is based on the small signal analysis. If we reorganize Eq. 7, we have:

$$
\left(\frac{n}{n^{\prime}}\right)^{2}\left(n^{\prime 2} \frac{C_{m}}{C_{0}}\right)+\left(\frac{n}{n^{\prime}}\right)-1=0
$$

When we solve the second order Eq. 8 and take the positive root, we can calculate the transformer ratio, $n$.

We utilize several methods to calculate $n^{\prime}$. First of these methods is the FEM simulations. The algorithm we use for the calculation of the forces is as follows. First we deflect the membrane to the operating point of the transducer. We apply a fixed bias voltage, $V_{d c}$ and perform a coupled field analysis on the FEM package ANSYS. As a second deflection, we apply a slightly larger voltage, $V_{d c}+V_{c o l} / 100$ and retrieve the force information on the membrane. The difference between the forces of two consecutive analysis is assumed to be the applied small signal force and $V_{c o l} / 100$ is the small signal voltage. The ratio of these two parameters is $n^{\prime}$.

One other way of calculating $n^{\prime}$ is using the deflection profile calculated in Section III.B. Recall that we can calculate the deflection of the membrane under a fixed bias voltage using Eq. 4. We can also calculate the electrostatic pressure, $P_{D C}$

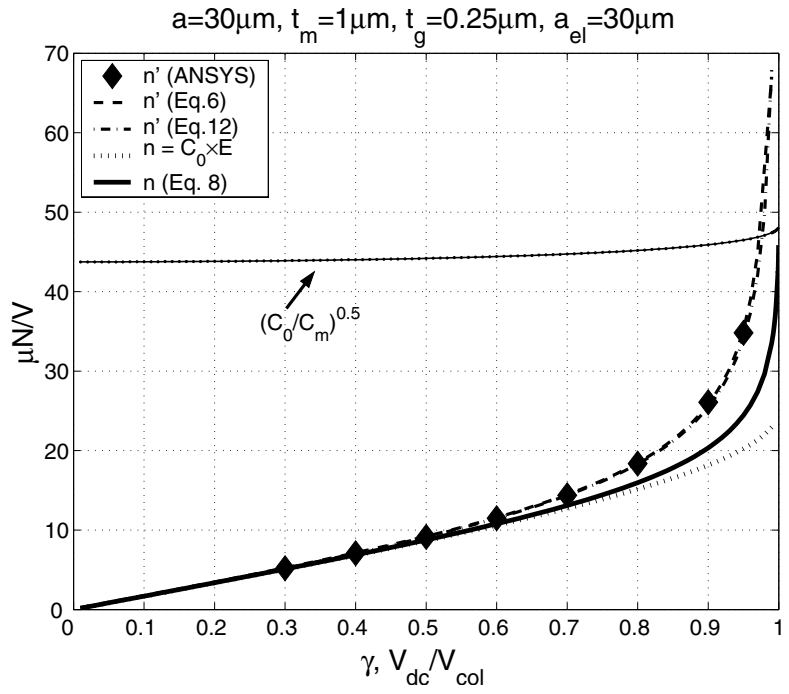

Fig. 3. $n^{\prime}$ values resulting from three different methods as a function of applied bias voltage(dashed, dash-dot, diamond). Calculated $n$ values from Eq. 8 (solid) and from capacitance-electric field multiplication (dotted) as a function of bias voltage(dotted).

acting on such a membrane as in [8]:

$$
\begin{array}{r}
P_{d c}(r)=\frac{\epsilon_{0} E_{d c}^{2}}{2}=\frac{\epsilon_{0} V_{d c}^{2}}{2\left(t_{g}-x(r)\right)^{2}} \\
F_{d c}=\int_{0}^{2 \pi} \int_{0}^{a_{e l}} P_{d c}(r) r d r d \theta \\
F_{a c}=\left.F_{d c}\right|_{V_{d c}}+V_{c o l / 100}-\left.F_{d c}\right|_{V_{d c}}
\end{array}
$$

where $E_{d c}$ is the electrical field in the gap. Once again, we calculate $n^{\prime}$ by taking the ratio of $F_{a c}$ and $V_{c o l} / 100$.

We also calculate $n^{\prime}$ using the method developed in [7]:

$n^{\prime}=2 \pi\left|\int_{0}^{a_{e l}} \frac{\epsilon_{0} V_{D C}}{\left(t_{g}-x(r)\right)^{2}}+\frac{\epsilon_{0} V_{D C}^{2}}{\left(t_{g}-x(r)\right)^{3}} \frac{\partial x(r)}{\partial V} d r\right|_{V=V_{D C}}$

In Eq. 12 the spring softening effect is not ignored, as long as the coupled field analysis is used during the calculation of deflection profile $x(r)$.

We calculate $n^{\prime}$ and $n$ values using the methods described ${ }^{1}$ for fixed membrane dimensions. Fig. 3 shows a summary of the comparison of the results for a single membrane size with full electrode coverage. Our analysis with FEM simulations can be extended to any membranes and electrode size. However, the numerical methods using Eq. 4 are only valid for thin plates.

Note that $n^{\prime}$ grows very fast and tends to increase indefinitely as the applied voltage approaches to the collapse voltage. We can see this behavior in Eq. 12. The term $\frac{\partial x(r)}{\partial V}$ stands for the sensitivity of the membrane to an applied voltage. The sensitivity of the membrane grows very fast when the bias voltage approaches to collapse. However, the correct transformer ratio does not diverge at the point of collapse. On the contrary, it has a finite maximum value at this point. Using

\footnotetext{
${ }^{1}$ Utilized material parameter values are: $Y_{0}=320 G P a$ and $\sigma=0.263$.
} 


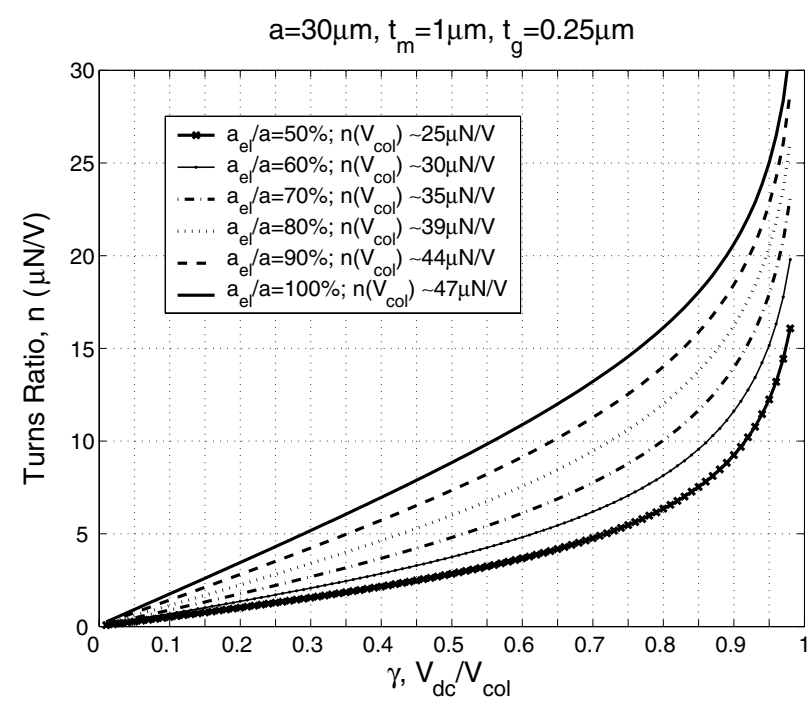

Fig. 4. Transformer ratio curves for the same membrane with different electrode sizes as a function of bias voltage.

Eq. 7 we can also determine this finite maximum analytically. At the point of the collapse, $n^{\prime}$ is infinite, therefore the denominator of the right hand side of the equation goes to zero. If we reorganize the expression, we find the maximum transformer ratio that can be achieved as follows:

$$
n\left(V_{c o l}\right)=\sqrt{\frac{C_{0}}{C_{m}}}
$$

Furthermore, if we utilize Mason's stiffness expression in 3, the maximum turns ratio for thin membranes with small gap heights approximately becomes:

$$
n\left(V_{c o l}\right) \simeq \sqrt{C_{0} \kappa}=\sqrt{\frac{16 \pi^{2} \epsilon_{0}\left(Y_{0}+T\right) t_{m}^{3}}{\left(1-\sigma^{2}\right) t_{g}}}
$$

Using the above equation, we calculate the maximum transformer ratio for the device in Fig. 3 as $44 \mu \mathrm{N} / \mathrm{V}$. This simple equation can be utilized to make quick calculations for the maximum transformer ratio value that can be achieved.

We investigated the behavior of the transformer ratio for membranes with different electrode sizes, using same approach. The resulting transformer ratio variation are plotted in Fig. 4.

\section{DiscusSiOn AND CONCLUSIONS}

Throughout this study, we assume that the transformer in the Mason's equivalent circuit is an ideal one. Therefore the transformer ratio value is assumed to be independent of the frequency up to the first parallel resonance frequency. We used a low frequency model to calculate the parameters. The simulations are performed at DC for several different approaches and compared to each other. We included the effect of spring softening capacitance in the equivalent circuit in order to calculate the transformer ratio accurately.

During the numerical calculations using MATLAB, we used the thin plate approximation for the deflected membrane. In addition we ignored the fringing fields in the calculations of the electric field in the gap. However, using ANSYS much more accurate results can be obtained for any membrane and electrode size and membrane material.

Note that we performed the calculations assuming that the mechanical port is terminated with vacuum environment. However, the transformer ratio is independent of the medium loading, if the medium pressure is small enough to ignore with respect to the DC bias force.

Using the information provided by Eq. 14, we can stress that the turns ratio is independent of the membrane size. The way of improving turns ratio is to increase the stiffness by using thicker membranes. Note that this analysis uses effective gap heights where the top electrode should be considered at the bottom of the membrane. In the case where the top electrode is at the top of the membrane the change of the effective gap height should also be taken into account since the transformation factor is also inversely proportional with $\sqrt{t_{g}}$.

The simulations performed for different electrode sized membranes show that higher transformation ratios can be achieved using larger electrodes. However this result will be further investigated by taking the coupling coefficient, $k_{T}$ into account.

There is a need to extend this analysis to any membrane size and material. Also methods are needed to measure the turns ratio experimentally.

\section{ACKNOWLEDGEMENTS}

The authors would like to thank Prof. Levent Degertekin from Georgia Institute of Technology for his valuable discussions. We also acknowledge the support of The Scientific and Technological Research Council of Turkey (TÜBITAK) in terms of the financial support through the research grant EEEAG\#105E023.

\section{REFERENCES}

[1] W. Mason, Electromechanical Transducers and Wave Filters. Van Nostrand, New York, 1942.

[2] F. Hunt, Electroacoustics: The Analysis of Transduction and Its Historical Background. American Institute of Physics for ASA, 1954.

[3] H. Koymen, M. N. Senlik, A. Atalar, and S. Olcum, "Parametric linear modelling of cMUT in vacuum," submitted to IEEE Trans. Ultrason., Ferroelect., Freq. Contr., 2006.

[4] A. Nikoozadeh, B. Bayram, G. G. Yaralioglu, and B. T. Khuri-Yakub, "Analytical calculation of collapse voltage of cMUT membrane," in Proc. of 2004 Ultrasonics Symposium, pp. 256-259, 2004.

[5] S. Timoshenko and S. Woinowsky-Krieger, Theory of Plates ans Shells New York: McGraw Hill, 1959.

[6] S. Olcum, M. N. Senlik, and A. Atalar, "Optimization of the gainbandwidth product of capacitive micromachined ultrasonic transducers," IEEE Trans. Ultrason., Ferroelect., Freq. Contr., vol. 52, pp. 2211-2219, 2005

[7] A. Caronti, E. Carotenuto, G. Caliano, and M. Pappalardo, "The effects of membrane metallization in capacitive microfabricated ultrasonic transducers," J. Acoust. Soc. Am. , vol. 115, pp. 651-657, 2004.

[8] A. Bozkurt, I. Ladabaum, A. Atalar, and B. T. Khuri-Yakub, "Theory and analysis of electrode size optimization for capacitive microfabricated ultrasonic transducers," IEEE Trans. Ultrason., Ferroelect., Freq. Contr., vol. 46, pp. 1364-1374, 1999. 